\title{
Structural Analysis of Helicobacter pylori VacA Reveals Insights into Oligomerization
}

Amanda L. Erwin ${ }^{1,2}$, Min Su ${ }^{1}$, Anne M. Campbell ${ }^{3}$, David Akey ${ }^{1}$, D. Borden Lacy ${ }^{4}$, Timothy L. Cover $^{3,4,5}$, and Melanie D. Ohi ${ }^{1,2^{*}}$

1. Life Sciences Institute, University of Michigan, Ann Arbor, MI, USA.

2. Department of Cell and Developmental Biology, University of Michigan, Ann Arbor, MI, USA.

3. Department of Medicine, Vanderbilt University School of Medicine, Nashville, TN, USA.

4. Department of Pathology, Microbiology, and Immunology, Vanderbilt University School of Medicine, Nashville, TN, USA.

5. Veterans Affairs Tennessee Valley Healthcare System, Nashville, TN, USA.

* Corresponding author: mohi@umich.edu

Helicobacter pylori is a Gram-negative bacterium that persistently colonizes the stomachs of $>50 \%$ of the human population, with a prevalence as high as $90 \%$ in developing nations [1, 2]. H. pylori infection causes gastritis and can lead to the development of peptic ulcer disease and gastric cancer in a subset of infected individuals [3, 4]. Gastric cancer is the third leading cause of cancer-related deaths worldwide and the World Health Organization has classified H. pylori as a type 1 carcinogen [5].

An important $H$. pylori virulence factor implicated in these diseases is the pore-forming toxin vacuolating cytotoxin A (VacA) $[6,7]$. VacA is named for its ability to induce vacuolation in cultured eukaryotic cells $[8,9]$. VacA has been reported to cause multiple cellular effects in addition to cellular vacuolation, including membrane permeabilization, mitochondrial dysfunction, cell death, autophagy, T cell inhibition, and other immunomodulatory effects [10].

VacA is secreted from H. pylori as an $88 \mathrm{kDa}$ monomer (p88), which shares very little sequence similarity to any characterized proteins from other bacterial species. $\mathrm{p} 88$ binds to the surface of gastric epithelial cells, oligomerizes, and forms anion-selective membrane channels [10]. p88 is comprised of two domains, an N-terminal p33 domain and a C-terminal p55 domain [11]. The p33 domain contains a hydrophobic region required for formation of the channel and regions within both the p33 and p55 domains mediate VacA oligomerization and binding to host cells [10]. Although most of the cellular effects of VacA are dependent on oligomerization, the underlying mechanism for how VacA oligomerizes is not understood.

To investigate the structural basis of VacA oligomerization, we analyzed VacA oligomers and VacA p88 monomers by single particle cryo-electron microscopy (cryo-EM) (Figure 1). Examining the oligomer particles by 2D classification with RELION revealed VacA oligomerizes into hexamers, heptamers, dodecamers, and tetradecamers [12]. We generated 3D reconstructions using RELION and cisTEM of VacA as a hexamer, heptamer, and dodecamer [12, 13]. Our highest resolution structure was of a VacA hexamer. Additionally, we generated a 3D reconstruction of a VacA p88 monomer using cryoSPARC [14]. Comparison of these structures enabled us to identify regions within the VacA p33 and p55 domains involved in oligomerization.

This study provides important insights into how VacA oligomerizes. Since the molecular 
mechanisms by which VacA elicits its variety of cellular responses are not fully elucidated, these structural studies will be used as springboard to understand VacA function within the context of cells [15].

References:

[1] PK Bardhan, Clin Infect Dis 25 (1997), p. 973.

[2] J Parsonnet, Infect Dis Clin North Am 12 (1998), p. 185.

[3] BJ Marshall and JR Warren, Lancet 1 (1984), p. 1311.

[4] S Suerbaum and P Michetti, N Engl J Med 347 (2002), p. 1175.

[5] IARC Monogr Eval Carcinog Risks Hum 61 (1994), p. 1.

[6] JC Atherton et al., J Biol Chem 270 (1995), p. 17771.

[7] TL Cover, MBio 7 (2016), p. e01869.

[8] RD Leunk et al., J Med Microbiol 26 (1988), p. 93.

[9] TL Cover et al., J Infect Dis 166 (1992), p. 1073.

[10] NJ Foegeding et al., Toxins (Basel) 8 (2016), p.

[11] JL Telford et al., J Exp Med 179 (1994), p. 1653.

[12] SH Scheres, J Struct Biol 180 (2012), p. 519.

[13] T Grant, A Rohou and N Grigorieff, Elife 7 (2018), p.

[14] A Punjani et al., Nat Methods 14 (2017), p. 290.

[15] This work was supported by a fellowship from the University of Michigan CDB Department to A.L.E.

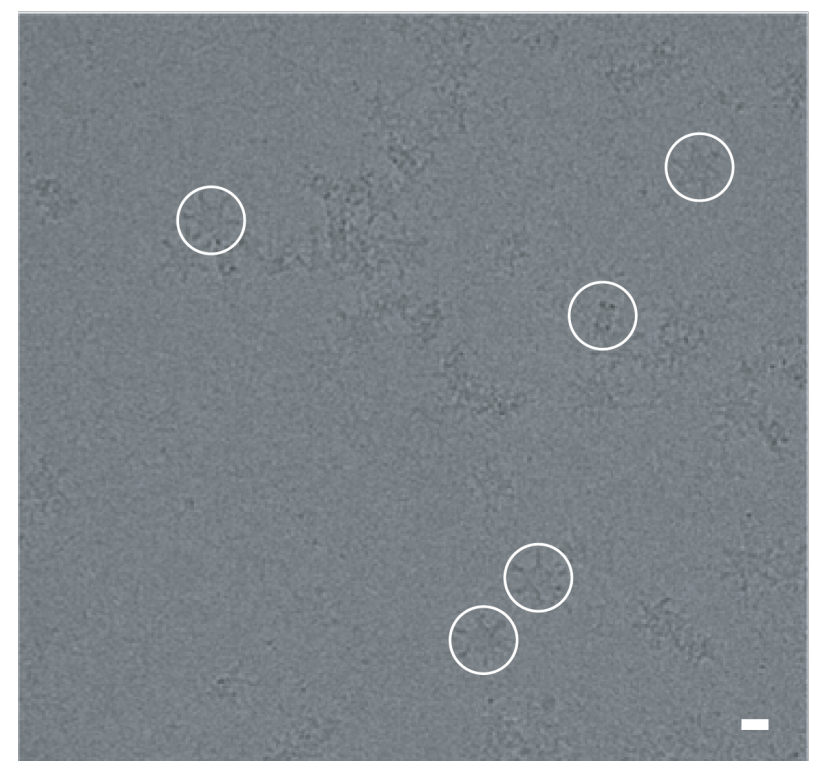

Figure 1. Cryo-EM analysis of VacA s1m1 oligomers. (A) Representative cryo-EM micrograph of VacA oligomer particles in vitreous ice. Some particles are highlighted with white circles. Scale bar $=$ $20 \mathrm{~nm}$. 